\title{
A Constrained Investment Policy for Defined - Contribution Pension Fund Management
}

\author{
Godswill U. Achi ${ }^{1} \&$ Okafor J. U. ${ }^{1}$ \\ ${ }^{1}$ Department of Mathematics, Abia State Polytechnic, Aba, Abia State, Nigeria \\ Correspondence: Godswill U. Achi, Department of Mathematics, Abia State Polytechnic, P.M.B. 7166, Aba, Abia \\ State, Nigeria. Tel: 234-803-705-8344. E-mail: achigods@yahoo.com
}

\author{
Received: December 25, 2012 Accepted: February 8, 2013 Online Published: March 12, 2013 \\ doi:10.5539/jmr.v5n2p6 URL: http://dx.doi.org/10.5539/jmr.v5n2p6
}

\begin{abstract}
This paper considered a stochastic control problem for the optimal management of a contribution pension fund model with solvency constraints. It is also strategic to accept the attitude of the fund manager who can invest in two assets: (a risky one and a non risky one in a standard Black Scholes market) and maximize the utility function consequent upon the current level of fund wealth. Our aim in this paper is to pose a constraint on the fund manager by ensuring that a solvency level is maintained on the fund wealth. This implies that the wealth of the running pension fund remains above a stipulated level i.e. the solvency level.
\end{abstract}

Keywords: stochastic control, stochastic optimal control, solvency level, defined-benefit, defined-contribution

MSC: 93E20, 91G80, 91G30, 60H35, 35R60

\section{Introduction}

A defined benefit pension plan is a scheme where the benefits have to do with employee's final remuneration and entitlement. If there is any contribution from the employee, it is predicted on the degree of remuneration which leaves the employer with the obligation of meeting his worker's financial benefits even on retirement. Thus, the employer documents these benefits for the onwards long-term funding via the pension schemes facility (Society of Actuaries in Ireland, 2003).

Usually, defined benefit pension schemes have ploughed a reasonable proportion of their assets in equities. The aim is to plough back some good financial returns with which the employer foots the employees' overall financial entitlements on a long-term basis.

Notably, the surplus returns accruing from equity investment come at a certain price. However, the risk involved with this price is that it comes at a foreseeable unstable rate of returns.

It, therefore, happens that there is the likelihood that such investment's volatility can rise at any given time causing a decrease in the worth of the company's assets, posing unmanageable liability chart to the employer and his company. Although there is no perfect gauge matching the fixed entitlement as regards the pension scheme, the uncertainty of stable returns can be reduced by investing in various available assets including bonds (Society of Actuaries in Ireland, 2003) .

It is strategic to accept the attitude of the fund manager who can invest in two assets (a risky one and a non risky one, in a standard Black and Scholes market) and maximize an inter temporal utility function consequent upon the current level of fund wealth (No Delaying).

The emphasis is posed on the constraints faced by the fund manager: by ensuring that a stipulated level is maintained on the fund wealth i.e. the solvency level, and the borrowing and short selling constraints on the allocation strategies. By implication, it is expedient that the wealth of the running pension fund stays above a stipulated levelthe solvency level.

\section{Formulation of the Model}

First, we state the assumptions before we present the model. 


\subsection{Assumptions of the Model}

We consider a continuous time financial market model which is:

(a) Competitive: i.e the investor optimizes his utility function on the whole time horizon;

(b) Frictionless: i.e all assets are divisible, no restriction on short sales, no transaction costs or taxes;

(c) The asset market contains no arbitrary opportunities: i.e there are neither free lunches nor lotteries;

(d) Default free: i.e. there is no disparity between interest rate for borrowing and lending;

(e) Continuously open: i.e. the investor can continuously trade in the market.

\subsection{The Mathematical Model of the Wealth Dynamics}

Let $(\Omega, \mathcal{F}, P)$ be a complete probability space with a filteration $\left\{\mathcal{F}_{t}\right\}_{t \geq 0}$ where $t \geq 0$ is the time variable. The filteration $\left\{\mathcal{F}_{t}\right\}_{t \geq 0}$ describing the information structure is generated by the trajectories of a 1-dimensional standard Brownian motion $\mathrm{B}(\mathrm{t}), \mathrm{t} \geq 0$, defined on the same probability space and completed with the addition of the null measure sets of $\mathcal{F}$.

The security market consists of two types of assets: riskless one, whose price $S^{0}(t), t \geq 0$ is governed by the dynamics

$$
\left\{\begin{array}{l}
d S^{0}(t)=r S^{0}(t) d t \\
S^{0}(0)=1
\end{array}\right.
$$

where $r \geq 0$ is the instantaneous spot rate of return and a risky asset, whose price dynamic $s^{1}(t), t \geq 0$ follows an Ito process and satisfies the stochastic differential equation

$$
\left\{\begin{aligned}
d S^{1}(t) & =\mu S^{1}(t) d t+\sigma S^{1}(t) d B(t), \\
S^{1}(0) & =S_{0}^{1}
\end{aligned}\right.
$$

where $\mu$ is the instantaneous rate of expected return and $\sigma>0$ is the instantaneous rate of volatility. We assume that the market assigns a premium for the risky investment i.e. $\mu>r$. The drift $\mu$ is given by the relation $\mu=r+\sigma \lambda$ where $\lambda>0$ is the instantaneous risk premium of the market assigns to the randomness expressed by the Standard Brownian motion $B(\cdot)$. The case $\lambda=0$ i.e. $\mu=r$ is trivial in a (natural) content of risk aversion.

Note: the interest rate is taken to be a constant while the solvency constraint is not considered.

Let $X(t), t \geq 0$ denote the state variable, and $\left\{\mathcal{F}_{t}\right\}_{t \geq 0}$-adapted process which represents the proportion of fund at any time.

The control variable, denoted by $\theta(t), t \geq 0$ is the $\left\{\mathcal{F}_{t}\right\}_{t \geq 0}$ progressively measurable process which represents the proportion of pension fund or accumulated wealth is invested in the risky asset. Therefore, the positivity of the wealth (due to the solvency constraints) and the borrowing and short selling constraints impose $\theta(t) \in[0,1]$ for every $t$. So the wealth dynamics satisfies

$$
\left\{\begin{array}{l}
d X(t)=\frac{\theta(t) x(t)}{S^{1}(t)} d S^{1}(t)+\frac{[1-\theta(t)] X(t)}{S^{0}(t)} d S^{0}(t)+c(t) d t-b(t) d t \\
X(o)=x_{0} \geq 0
\end{array}\right.
$$

where $\frac{\theta(t) X(t)}{S^{1}(t)}$ and $\frac{[1-\theta(t)] X(t)}{S^{0}(t)}$ are the quantities in the portfolio of the risky asset and the riskless asset respectively, the non-negative process $c(t), t \geq 0 ; b(t), t \geq 0$ represents the flow of contributions and benefits respectively.

The wealth dynamics (2.1) can be written in the following way

$$
\left\{\begin{array}{l}
d X(t)=[(r+\sigma \lambda \theta(t)) X(t))+c(t)-b(t)] d t+\sigma \theta(t) X(t) d B(t) t \geq 0 \\
X(0)=x
\end{array}\right.
$$

As mention earlier, the solvency constraint must be respected. More precisely the process $x(\cdot)$ describing the fund wealth is subject to the following constraint.

$$
X(t) \geq l(t) \quad p: a s \forall t \geq 0
$$

where the non-negative deterministic function $l(t), t \geq 0$ represents the solvency level. 


\subsection{Contributions}

In the population stationary, the contribution rate $c(\cdot)$ is exogenous. We assume that the workers who enter into the pension fund are of a homogeneous class. Moreover, we suppose that their entrance flow is constant on time and that each participant adheres to a length of time represented to an exogenous constant $T>0$. Due to these demographic assumptions, the aggregate contribution flow increases linearly on time in the interval $[0, T]$ and is equal to a constant $c>0$ after time $T$. We can imagine that each member who is adhering to the fund pays to the fund a contribution rate equal to $\alpha \omega$, where $\alpha \epsilon(0,1)$ and where $\omega>0$ is the (constant) wage rate earned by each member. Then, denoting by $\bar{c}$ the entrance flow of people into the fund, we can write the flow of aggregate contribution as

$$
C(t)= \begin{cases}\alpha \omega \cdot \bar{c} t, & 0 \leq t \leq T \\ \alpha \omega \cdot \bar{c} T, & t>T\end{cases}
$$

\subsection{Benefits}

Due to the demographic stationarity, again, we assume that the flow of benefits starts at time $T$, when the first retirements occur, and that, after that date, it is given by a constant $g$ representing the minimum guarantee flow. We assume that $g \geq c$, because $\mathrm{g}$ has to be in some way, the capitalization of the contributions paid by the members who are retired. For instance, we can imagine that the fund pays to the generic member in retirement as (Lump sum) minimum guarantee the capitalization at a minimum guaranteed rate $\delta \epsilon[o, r]$ of the contribution paid by him in the interval during which he was adhering to the fund. In this case, coherently with Equation (2.4), we can write the aggregate minimum guarantee flow, for $t \geq T$ as

$$
g=\bar{c} \int_{t-T}^{t}(\alpha \omega) e^{\delta(t-u)} d u
$$

i.e.

$$
g= \begin{cases}c, & \text { if } \delta=0 \\ \bar{c} \cdot(\alpha \omega) \frac{e^{\delta T}-1}{b}, & \text { if } \delta>0 .\end{cases}
$$

\subsection{Solvency Level}

We can now control the behavior of the fund manager to avoid unruly behavior and to ensure that the fund is capable of paying at least the desire benefits at each time $t \geq 0$ by maintaining a solvency level. Without enforcing this constraint the fund manager may implore strategies that may fall out with the social goal of the pension fund. Hence, we let the solvency level $l(\cdot)$ imposed in (2.3) be a non-decreasing continuous function, which is constant after time $T$.

\subsubsection{Assumptions of the Solvency Level}

i) At the beginning the company should hold a given minimum startup level $l_{0}=l(0) \geq 0$;

ii) For $t \in[0, T]$, the solvency level is the capitalization at a rate $\beta \leq r$ of initial minimum wealth $l_{0}$ and of the aggregate contributions paid up to time $t$, therefore

$$
l(t)=l_{0} e^{\beta t}+\int_{0}^{t} \alpha \omega \cdot \bar{c} s e^{\beta(t-s)} d s .
$$

iii) After time T the solvency level is constant, i.e. $l(t)=l=l(T)$ for $t \geq T$.

The rate $\beta$ could be chosen, for example by an authority with respect to the market's parameter.

\section{The Optimization Problem}

We consider a finite horizon optimization problem in the interval $[0, T]$ related to an objective functional with this form

$$
\mathbb{E}\left[\int_{0}^{T} e^{-\rho t} U(t, X(t)) d t+f(X(T))\right]
$$

Here $\rho>0$ is the individual discount factor of the manager and $U$ is the utility function.

Assumptions 1.

A. The current utility function $U$ is such that 
i) $U$; $C \rightarrow R$ has the structure $U(s, x)=u(x-l(s))$, where $u \rightarrow[0,+\infty) \rightarrow R$;

ii) $u \in C([0,+\infty) ; R)$;

iii) $u$ is increasing and concave.

B. The exit/bequest utility function $f$ is such that

i) $f \in C([l(T),+\infty) ; R)$;

ii) $f$ is increasing and concave.

Remark 1 The utility functions $U$ and $f$ are defined where the wealth process $X($.$) must live; all the functions of$ the form $(x)=\frac{\left(x-x_{0}\right)^{r}}{r}$, for $x_{0} \leq 0, r \in(0,1)$ always give rise to functions $U$ satisfying A.

All the functions of the form $f(x)=\frac{\left(x-x_{0}\right)^{r}}{r}$, for $x_{0} \leq 0 . r \in(0,1)$ satisfy B.

For $(s, x) \in C$, the problem consists in maximizing over the set of the admissible strategies.

Hence, the wealth process $X(t)$ is governed by

$$
\left\{\begin{array}{l}
d X(t)=r X(t) d t+k t d t+\sigma \theta(t) X(t) d \bar{B}^{S}(t) \\
X(s)=l(s)
\end{array}\right.
$$

where, by Girsanov's Theorem, the process $\bar{B}^{s}(\cdot)=B^{S}(\cdot)+\lambda(\cdot-s)$ is a Brownian motion under the probability $\tilde{P}=\exp \left(-\lambda B^{s}(T)-\frac{\lambda^{2}}{2}(T-s)\right) . P$ in the interval $[s, T]$.

Since $X \in C\left([s, T] ; L^{P}(\Omega, \mathrm{P})\right)$ for any $P \geq 1$, by Holder's inequality it also holds

$$
\widetilde{\mathbb{E}}\left[\int_{S}^{T}|X(t)|^{2} d t\right]<+\infty
$$

So that

$$
\widetilde{\mathbb{E}}\left[\int_{s}^{t} X(\gamma) d B^{s}(\gamma)\right]=0, \text { for all } t \in[s, T] .
$$

Proposition 1 Suppose that assumptions $A$ and B hold true. Then there exists a constant $C>0$ such that $V(s, x) \leq$ $C(1+x)$ for all $(s, x) \in C$.

Proof. Let $(s, x) \in C$. By assumptions A and B there exists $C>0$ such that $U(t, y) \leq C(1+y)$ for any $t \in[s, T], y \geq$ $l(t)$ and $\geq f(y) \leq C(1+y)$ for any $y \geq l(t)$. Let $\theta(\cdot) \in \ominus_{a d}(s, x)$, then setting $X(t)=X(t, s, x, \theta(\cdot))$ we have

$$
E\left[\int_{S}^{T} e-^{\rho t}[U(t, X(t))] d t+f(X(T))\right] \leq C E\left[\int_{S}^{T} e-^{\rho t}(1+X(t) d t+(1+X(T))]\right.
$$

Taking into account that $X \in C([S, T]) ; L^{2}(\Omega)$

We have

$$
\mathbb{E}\left[\int_{s}^{t} \theta(r) X(r) d B^{S}(r)\right]=0, \quad \forall t \in[S, T]
$$

Such that

$$
\left\{\begin{array}{l}
d \mathbb{E}[X(t)]=r \mathbb{E}[X(t) d t+k t d t]+\sigma \lambda \mathbb{E}[\theta(t) X(t)] d t \leq(r+\sigma \lambda) \mathbb{E}[X(t)] d t+k T d t \\
\mathbb{E}[X(s)]=x
\end{array}\right.
$$

hence, for some $C>0$

$$
\mathbb{E}[X(t)] \leq\left(x+\frac{K T}{r+\sigma \lambda}\right) e^{(r+\sigma \lambda)(t-s)}-\frac{K T}{r+\sigma \lambda} \leq C(1+x)
$$

The estimate (3.4) does not depend on the control, thus the claim follows putting (3.4) into (3.3) and taking the supremum over $\theta(\cdot) \in \ominus_{a d}(s, x)$.

Proposition 2 Let $s \in[0, T]$; the function $x \rightarrow V(s, x)$ is concave on $[C(s),+\infty]$. 
Proof. Fix $x, x^{1} \geq l(s)$; for $\gamma \in[0,1]$, set $x_{\gamma}:=\gamma x+(1-\gamma) x^{1}$; of course $x_{\gamma} \geq l(s)$. we have to prove that

$$
V\left(s, x_{\gamma}\right) \geq \gamma V(s, x)+(1-\gamma) V\left(s, x^{1}\right) .
$$

Take $\theta(\cdot) \in \ominus_{a d}(s, x)$ and $\theta^{1}(\cdot) \in \ominus_{a d}\left(s, x^{1}\right) \varepsilon$-optimal for $x, x^{1}$ respectively and $X(\cdot), X^{1}(\cdot)$ the corresponding trajectories; then

$$
\begin{aligned}
& \gamma V(s, x)+(1-\gamma) V\left(s, x^{1}\right) \\
\leq & \gamma[J(s, x ; \theta(\cdot))+\varepsilon]+(1-\gamma)\left[J\left(s, x^{1} ; \theta^{1}(\cdot)\right)+\varepsilon\right] \\
= & \varepsilon+\gamma^{J}(s, x ; \theta(\cdot))+(1-\gamma) J\left(s, x^{1} ; \theta^{1}(\cdot)\right) \\
= & \varepsilon+\gamma \mathbb{E}\left[\int_{S}^{T} e^{-\rho t} U(t, X(t)) d t+f(X(T))\right]+(1-\gamma) \mathbb{E}\left[\int_{S}^{T} e^{-\rho t} U\left(t, X^{1}(t)\right) d t+f\left(X^{1}(T)\right)\right] \\
= & \mathbb{E}\left[\int_{S}^{T} e^{-\rho t}\left[\gamma U(t, X(t))+(1-\gamma) U\left(t, X^{1}(t)\right)\right] d t\right]+\mathbb{E}\left[\gamma f(X(T))+(1-\gamma) f\left(X^{\prime}(T)\right)\right]+\varepsilon .
\end{aligned}
$$

The concavity of $u, f$ implies that

$$
\begin{gathered}
\gamma U(t, X(t))+(1-\gamma) U\left(t, X^{\prime}(t)\right) \leq U\left(t, \gamma X(t)+(1-\gamma) X^{\prime}(t)\right), \quad \forall t \in[s, T], \\
\gamma f(X(t))+(1-\gamma) f\left(X^{\prime}(t)\right) \leq f\left(\gamma X(t)+(1-\gamma) X^{\prime}(t)\right), \quad \forall t \in[s, T] .
\end{gathered}
$$

Consequently, setting

$$
X_{\gamma}(\cdot):=\gamma X(\cdot)+(1-\gamma) X^{\prime}(\cdot)
$$

we obtain

$$
\gamma V(s, x)+(1-\gamma) V\left(s, x^{\prime}\right) \leq \varepsilon+\mathbb{E}\left[\int_{S}^{T} e^{-\rho t} U\left(X_{\gamma}(t)+f\left(X_{\gamma}(T)\right)\right] .\right.
$$

If there exists $\theta_{\gamma}(\cdot) \in \ominus\left(s, x_{\gamma}\right)$ such that $X_{\gamma}(\cdot) \leq X\left(\cdot ; s, x_{\gamma}, \theta_{\gamma}(\cdot)\right)$, then we would have

$$
\varepsilon+\mathbb{E}\left[\int_{S}^{T} e^{-\rho t} U\left(X_{\gamma}(t)\right) d t+f\left(X_{\gamma}(T)\right)\right] \leq \varepsilon+J\left(s, x_{\gamma} ; \theta_{\gamma}(.)\right) \leq \varepsilon+V\left(s, x_{\gamma}\right),
$$

i.e.

$$
\gamma V(s, x)+(1-\gamma) V\left(s, x^{\prime}\right) \leq \varepsilon+V\left(s, x_{\gamma}\right)
$$

and therefore, by the arbitrariness of $\varepsilon$, the claim (3.5) would be proved. We will show that

$$
\theta_{\gamma}(t):=\alpha(t) \theta(t)+d(t) \theta^{\prime}(t),
$$

Where

is good. The admissibility of $\theta_{\gamma}(\cdot)$ is clear since;

$$
\alpha(\cdot)=\gamma \frac{X(\cdot)}{X_{\gamma}(\cdot)}, d(\cdot)=(1-\gamma) \frac{X^{\prime}(\cdot)}{X_{\gamma}(\cdot)},
$$

i) For any $t \in[s, T]$ we have $\theta(t), \theta^{1}(t) \in[0,1]$, and $\alpha(t)+d(t)=1$, so that by convexity of $[0,1]$ we get $\theta_{\gamma}(t) \in[0,1]$

ii) By construction $X_{\gamma}(t) \geq l(t)$ for any $t \in[s, T]$.

Note that actually we will prove that $X_{\gamma}(\cdot)=X\left(\cdot ; s, x_{\gamma}, \theta_{\gamma}(\cdot)\right)$. The equation satisfied by $X_{\gamma}(\cdot)$ in the interval $[s, T]$ is

$$
\begin{aligned}
& d X_{\gamma}(t)=\gamma d X(t)+(1-\gamma) d X^{\prime}(t) \\
= & \gamma\left[[(r+\sigma \lambda \theta(t)) X(t)+k t] d t+\sigma \theta(t) X(t) d B^{s}(t)\right]+(1-\gamma)\left[\left[\left(r+\sigma \lambda \theta^{\prime}(t)\right) X^{\prime}(t)+k d\right] d t+\sigma \theta^{\prime}(t) X^{\prime}(t) d B^{s}(t)\right] \\
= & {\left[r X_{\gamma}(t)+\sigma \lambda\left(\gamma \theta(t) X(t)+(1-\gamma) \theta^{\prime}(t) X^{\prime}(t)\right)+k t\right] d t+\sigma\left[\gamma \theta(t) X(t)+(1-\gamma) \theta^{\prime}(t) X^{\prime}(t)\right] d B^{s}(t) } \\
= & {\left[r X_{\gamma}(t)+k t\right] d t+\sigma \lambda\left[\gamma \theta(t) \frac{X(t)}{X_{\gamma}(t)}+(1-\gamma) \theta^{\prime}(t) \frac{X^{\prime}(t)}{X_{\gamma}(t)}\right] X_{\gamma}(t) d t+\sigma\left[\gamma \sigma(t) \frac{X(t)}{X_{\gamma}(t)}(1-\gamma) \theta^{\prime}(t) \frac{X^{\prime} 1(t)}{X_{\gamma}(t)}\right] X_{\gamma}(t) d B^{s}(t) } \\
= & {\left[\left(r+\sigma \lambda \theta_{\gamma}(t)\right) X_{\gamma}(t)+k t\right] d t+\sigma \theta_{\gamma}(t) X_{\gamma}(t) d B^{s}(t) . }
\end{aligned}
$$


and the claim follows.

\section{Dynamic Programming}

We adopt a dynamic programming approach to study the optimization problem. The core of the dynamic programming is the so-called dynamic programming principle, which in our context can be stated as follows.

Theorem The value function $V$ satisfies the dynamic programming equation, i.e. for every $s \in[0, T], x \in$ $[l(s),+\infty)$ and for any family of stopping times $\left(\tau^{\theta(\cdot)}\right)_{\theta(\cdot) \in \ominus_{a d}(s, x)}$ taking values in $[s, T]$, the following functional equation holds true:

$$
V(s, x)={ }_{\theta(\cdot) \in \ominus a d(s,)}^{S U P} E\left[\int_{S}^{T} e^{-\rho t} U(t, X(t ; s, x, \theta(\cdot))) d t+V\left(\tau^{\theta(\cdot)}, X\left(\tau^{\theta(\cdot)} ; s, x, \theta(\cdot)\right)\right)\right] .
$$

Proof. Actually we give here only a heuristic proof. For simplicity of notation we suppress the possible dependence of $\tau$ on $\theta(\cdot)$, i.e. we will write.

Simply $\tau$ to intend $\tau^{\theta(\cdot)}$. Therefore, we have

$$
\begin{aligned}
V(s, x) & ={ }_{\theta(\cdot) \in \Theta_{a d}(s, x)}^{S U P} \mathbb{E}\left[\int_{s}^{T} e^{-\rho t} U(t, X(t ; s, x, \theta(\cdot))) d t\right] \\
& ={ }_{\theta(\cdot) \in \Theta_{a d}(s, x)}^{S U P} \mathbb{E}\left[\int_{s}^{\tau} e^{-\rho t} U(t, X(t ; s, x, \theta(\cdot))) d t+\int_{\tau}^{T} e^{-\rho t} U(t, X(t ; s, x, \theta(\cdot))) d t\right] \\
& \left.\left.={ }_{\theta(\cdot) \in \Theta_{a d}(s, x)}^{S U U} \mathbb{E}\left[\int_{s}^{\tau} e^{-\rho t} U(t ; s, x, \theta(\cdot))\right) d t+\mathbb{E}\left[\int_{\tau}^{T} e^{-p t} U(t ; s, x, \theta(\cdot))\right) d t \mid F_{T}^{s}\right]\right] \\
& \left.={ }_{\theta(\cdot) \in \Theta_{a d}(s, x)}^{S U P} \mathbb{E}\left[\int_{s}^{\tau} e^{-\rho t} U(X(t ; s, x, \theta(\cdot))) d t+J(\tau ; s, x, \theta(\cdot))\right)\right] \\
& \leq_{\theta(\cdot) \in \Theta_{a d}(x)}^{S U P} \mathbb{E}\left[\int_{s}^{\tau} e^{-\rho t} U(X(t ; s, x, \theta(\cdot))) d t+V(t, X(\tau ; s, x, \theta(\cdot)))\right] .
\end{aligned}
$$

Conversely, for fixed $\varepsilon>0$, for any $\left(s^{\prime}, y\right)$ such that $s^{\prime} \in[s, T], y \geq l\left(s^{\prime}\right)$, let $\theta_{s^{\prime}, y}^{\varepsilon}(\cdot)$ a control $\varepsilon$-optimal for the pair $\left(s^{\prime}, y\right)$, i.e $J\left(s^{\prime}, y ; \theta_{s^{1}, y}^{\varepsilon}(\cdot)\right) \geq V\left(s^{\prime}, y\right)-\varepsilon$. Let $\theta(\cdot) \in \ominus_{a d}(s, x)$ and define the control

$$
\bar{\theta}(t)= \begin{cases}\theta(t), & \text { if } t \in[s, T], \\ \theta_{\tau, X(\tau ; s, x, \theta(\cdot))}^{\varepsilon}(t), & \text { if } t \in[\tau, T] .\end{cases}
$$

Therefore we have $\bar{\theta}(\cdot) \in \ominus_{\text {ad }}(s, x)$, such that;

$$
\begin{aligned}
V(s, x) & \geq J(s, x ; \bar{\theta}(\cdot))=\mathbb{E}\left[\int_{s}^{\tau} e^{-\rho t} U(t, X(t ; s, x, \theta(\cdot))) d t\right]+\mathbb{E}\left[\int_{\tau}^{T} e^{-\rho t} U\left(t, X(\tau ; s, x, \theta(\cdot)) ; \theta_{\tau, X(\tau ; s, x, \theta(\cdot))}^{\varepsilon}\right) d t\right] \\
& =\mathbb{E}\left[\int_{s}^{\tau} e^{-\rho t} U(t, X(t ; x, \theta(\cdot))) d t+\mathbb{E}\left[\int_{\tau}^{T} e^{-\rho t} U\left(t, X\left(t, \tau, X\left(\tau ; x, s, \theta(\cdot) \theta_{\tau, X(\tau ; s, x, \theta(\cdot))}^{\varepsilon}\right) d t \mid \mathcal{F}_{\tau}^{s}\right)\right]\right]\right. \\
& \left.=\mathbb{E}\left[\int_{s}^{\tau} e^{-\rho t} U(t, X,(t ; T, x, \theta(\cdot))) d t+J\left(\tau, X(\tau ; s, x, \theta(\cdot)) ; \theta_{\tau, X(\tau ; s, r ; \cdot(\cdot))}^{\varepsilon} \cdot\right)\right)\right] \\
& \geq \mathbb{E}\left[\int_{s}^{\tau} e^{-\rho t} U(t, X(t ; s, x, \theta(\cdot))) d t+V(\tau, X(\tau ; s, x, \theta(\cdot)))\right]-\varepsilon .
\end{aligned}
$$

By arbitrariness of $\varepsilon$ and taking the supremum over all $\theta(\cdot) \in \ominus_{a d}(s, x)$, we have the desired inequality and so the claim.

However, we want to point out that we have proved the continuity of our value function. The inequality

$$
\left.V(s, x) \geq \int_{s}^{T} e^{-\rho t} U(t, X(t ; s, x, 0)) d t+V\left(s^{\prime} ; s, x, 0\right)\right), \quad 0 \leq s \leq s^{\prime} \leq T, x \geq l(s) .
$$

Which can be proved without any measurable selection argument, because in this case we are on a deterministic trajectory? Therefore we can use the argument of (Yong \& Zhou, 1999) in order to prove the dynamic programming principle without loss of generality.

\subsection{The HJB Equation}

We can now introduce the following Hamiltonian function associated with our problem 


$$
\mathcal{H}(s, x, p, Q):={ }_{\theta \in[0,1]}^{S U P} \mathcal{H}_{c v}(s, x, p, Q ; \theta), s \in[0, T], x \in[l(s),+\infty), p, Q \in \mathbb{R},
$$

where

$$
\mathcal{H}_{c v}(s, x, p, Q ; \theta):=e^{-\rho s} U(s, x)+[(r+\sigma \lambda \theta) x+k s] p+\frac{1}{2} \sigma^{2} \theta^{2} x^{2} Q .
$$

The HJB equation on the domain $c$ formally associated with our problem is

$$
\left\{\begin{array}{l}
-v_{s}(s, x)-\mathcal{H}\left(s, x, v_{x}(s, x), v_{x x}(s, x)\right)=0,(s, x) \in{ }^{\circ} \mathrm{C} \\
v(T, x)=f(x), x \in[l(T),+\infty)
\end{array}\right.
$$

Setting

We now have

$$
\mathcal{H}_{c v}^{0}(x, p, Q ; \theta):=\sigma \lambda \theta x p+\frac{1}{2} \sigma^{2} \theta^{2} x^{2} Q
$$

$$
\mathcal{H}(s, x, p, Q)=e^{-\rho s} U(s, x)+(r x+k s) p+{ }_{\theta \in[0,1\rceil}^{S U P} \mathcal{H}_{c v}^{0}(x, p, Q ; \theta) .
$$

To evaluate the Hamiltonian we can observe that the function

$$
\mathcal{H}_{c v}^{0}(x, p, Q ; \theta)=\sigma \lambda \theta x p+\frac{1}{2} \sigma^{2} \theta^{2} x^{2} Q
$$

When $p \geq 0, Q \leq 0, p^{2}+Q^{2}>0$, has a unique maximum point over $\theta \in[0,1]$ given by

$$
\theta^{*}=\left(-\frac{\lambda p}{\sigma x Q}\right) \wedge 1
$$

Where we meant that, for $Q=0$, it is $\theta^{*}=1$ and

$$
\mathcal{H}^{0}(x, p, Q ; \theta):={ }_{\theta \in[0,1]}^{S U P} \mathcal{H}_{c v}^{0}(x, p, Q ; \theta)=\left\{\begin{array}{l}
-\frac{\lambda^{2} p^{2}}{2 Q}, \text { if } \theta^{*}<1 \\
\sigma \lambda x p+\frac{1}{2} \sigma^{2} \theta^{2} x^{2} Q, \text { if } \theta^{*}=1 .
\end{array}\right.
$$

When $p=Q=0$ each $\theta \in[0,1]$ is a maximum point of $\mathcal{H}_{c v}^{0}$ and $\mathcal{H}^{0}(x, 0,0)=0$.

\subsection{An Explicit Solution}

We want to show in this section how the problem can be solved in close form when some constraints on the parameters and a special form of $u$ and $f$ are considered. We let $\gamma \in(0,1)$ and assume that

$$
\begin{cases}\text { (i) } & u(y)=\frac{y^{\gamma}}{\gamma}, y \geq 0 . \\ \text { (ii) } & f(x)=k \frac{(x-l(T))^{\gamma}}{\gamma}, x \geq l(T), k \geq 0 . \\ \text { (iii) } & \beta=r . \\ \text { (iv) } & \lambda \leq \sigma(1-\gamma) .\end{cases}
$$

Following (Merton, 1969, 1971) we look for a solution to equation of the form

$$
v(s, x)=C(s) e^{-\rho t} \frac{(x-l(s))^{\gamma}}{\gamma},(s, x) \in C .
$$

We have, for $(t, x) \in C \backslash \partial^{*} C$ (by the symbols $v_{s}(0, x), v_{s}(T, x)$ we respectively mean $v_{s}\left(0^{+}, x\right), v_{s}\left(T^{-}, x\right)$,

$$
\left\{\begin{array}{l}
v_{s}(s, x)=-C(s) \rho e^{-\rho s}\left[\frac{(x-l(s))^{\gamma}}{\gamma}+l^{\prime}(s)(x-l(s))^{\gamma-1}\right]+C^{\prime}(s) e^{-\rho s} \frac{(x-l(s))^{\gamma}}{\gamma}, \\
v_{x}(s, x)=C(s) e^{-\rho s}(x-l(s))^{\gamma-1}, \\
v_{x x}(s, x)=C(s) e^{-\rho s}(\gamma-1)(x-l(s))^{\gamma-2} .
\end{array}\right.
$$

Note that, for $(s, x) \in C \backslash \partial^{*} C$,

$$
-\frac{\lambda v_{X}(s, x)}{\sigma x v_{x x}(s, x)}=\frac{\lambda}{\sigma(1-\gamma)} \cdot \frac{(x-l(s))}{x} \leq 1,
$$


So that, for $(s, x) \in C \backslash \partial^{*} C$,

$$
\mathcal{H}^{0}\left(x, v_{x}(s, x), v_{x x}(s, x)\right)=-\frac{\lambda^{2} v_{x}(s, x)^{2}}{2 v_{x x}(s, x)}=C e^{-\rho s} \frac{\lambda^{2}}{2(1-\gamma)} \cdot(x-l(s))^{\gamma} .
$$

Putting the expressions for the derivation of $v$, we get, taking into account that $l^{\prime}(s)+k s$,

$$
\left[\frac{1}{\gamma} C^{\prime}(s)+C(s)\left[\frac{\rho}{\gamma}-r-\frac{\lambda^{2}}{2(1-\gamma)}\right]-\frac{1}{\gamma}\right](x-l(s))^{\gamma}=0 .
$$

Therefore, if $C(s)$ is the unique solution to the ordinary differential equation

$$
\left\{\begin{array}{l}
C^{\prime}(s)+\left[\rho-\gamma r-\frac{\lambda^{2} r}{2(1-\gamma)}\right] C(s)=0, \\
C(T)=k e^{\rho T}
\end{array}\right.
$$

then

$$
v(s, x)=C(s) e^{-\rho s} \frac{(x-l(s))^{\gamma}}{\gamma},(s, x) \in C,
$$

is a classical solution on $C \backslash \partial^{*} C$. Moreover such $v$ satisfies the lateral boundary condition

$$
v(s, l(s))=0, s \in[0, T]
$$

and the terminal boundary condition

$$
v(T, x)=f(x), \quad x \geq l(T) .
$$

Note that condition (iv) $\lambda \leq \sigma(1-\gamma)$, guarantees that the maximum point in the Hamiltonian is smaller than 1, hence the no borrowing constraint is never active: this allows to keeping $\widetilde{\mathcal{H}}^{0}$ in the form which is suitable to find the explicit solution.

\section{Conclusion}

This paper considered the attitude of the fund manager who can invest in two assets; a risky one and a non risky one, in a standard Black-Scholes market and maximize the utility function consequent upon the current level of fund wealth. We propose that the wealth of the running pension fund remains above a stipulated level which is regarded as the solvency level.

\section{References}

Boulier, J. F, Huang, S. J., \& Taillard, G. (2001). Optimal management under stochastic interest rates: the case of a protected pension fund. Insurance: Mathematics and Economics, 28(2), 173-189. http://dx.doi.org/10.1016/S0167-6687(00)00073-1

Cadenillas, A., \& Sethi, S. (1997). Consumption-investment problem with subsistence consumption, bankruptcy and random market coefficients. Journal of Optimization Theory and Applications, 93(2), 243-272. http://dx.doi.org/10.1023/A:1022640321499

Cairns, A. J. G., Blake, D., \& Dowd, K. (2000). Optimal dynamics asset allocation for defined-contribution pension plans. Proceedings of the 10 $0^{\text {th }}$ AFIR International Colloquium, 131-154.

Choulli, T., Taksar, M., \& Zhou, X. Y. (2003). A diffusion model for optimal dividend distribution for a company with constraints on risk control. SIAM Journal on Control and Optimization, 41(6), 1946-1979. http://dx.doi.org/10.1137/S0363012900382667

Deelstra, G., Grasselli, M., \& Koehl, P. F. (2003). Optimal investment strategies in the presence of a minimum guarantee. Insurance: Mathematics and Economics, 33(1), 189-207. http://dx.doi.org/10.1016/S0167-6687(03)00153-7

Di Giacinto, M., Federico, S., \& Gozzi, F. (2008). Optimal management of pension funds: a stochastic control approach. "RICAM workshop on Optimization and Optimal Control" Linz.

Di Giacinto, M., Federico, S., \& Gozzi, F. (2011). Pension funds with a minimum guarantee: a stochastic control approach. Finance and Stochastics, 15(2), 297-342. http://dx.doi.org/10.1007/s00780-010-0127-7 
El Karoui, N., Jeanblanc, M., \& Lacoste, V. (2005). Optimal portfolio management with American capital guarantee. Journal of Economics and Dynamic Control, 29(3), 449-468. http://dx.doi.org/10.1016/j.jedc.2003.11.005

Federico, S. (2008). A pension fund in the accumulation phase: a stochastic control approach. Banach Center publications, 83: Advances in Mathematics of Finance.

Haberman, S., \& Vigna, E. (2001). Optimal investment strategy for the defined contribution pension schemes. Insurance: Mathematics and Economics, 28(1), 232-262.

Haberman, S., \& Vigna, E. (2002). Optimal investment strategies and risk measure in defined contribution pension schemes. Insurance: Mathematics and Economics, 31(1), 35-69. http://dx.doi.org/10.1016/S0167-6687(02)00128-2

Karatzas, I., Lehoczky, J. P., Sethi, S., \& Shreve, S. E. (1986). Explicit solution of a general consumption/investment problem. Mathematics of Operations Research, 11(2), 261-294. http://dx.doi.org/10.1287/moor.11.2.261

Merton, R. C. (1969). Lifetime portfolio selection under uncertainty: the continuous-time case. Review of Economics and Statistics, 51, 247-257. http://dx.doi.org/10.2307/1926560

Merton, R. C. (1971). Optimal consumption and portfolio rules in a continuous-time model. Journal of Economics Theory, 3, 373-413. http://dx.doi.org/10.1016/0022-0531(71)90038-X

Sethi, S., \& Taksar, M. (1992). Infinite-horizon investment consumption model with a nonterminal bankruptcy. Journal of Optimization Theory and Applications, 74(2), 333-346. http://dx.doi.org/10.1007/BF00940898

Sethi, S., Taksar, M., \& Presman, E. (1992). Explicit solution of a general consumption/portfolio problem with subsistence consumption and bankruptcy. Journal of Economic Dynamics and Control, 16(3-4), 747-768. http://dx.doi.org/10.1016/0165-1889(92)90056-K

Soner, H. M. (2004). Stochastic Optimal control in finance, Cattedra Galileiana, Pisa: Scuola Normale Superiore.

The Society of Actuaries in Ireland. (2003). Current Funding Issues for Defined Benefit Pension Schemes. Retrieved from https://dx.doi.web.actuaries.i.e.Current_Funding_Issues_for_Defined_Benefit_Pension_Schemes

Youg, J., \& Zhou, X. Y. (1999). Stochastic controls: Hamiltonian systems and HJB equations. New York: Springer Verlag.

Zariphopoulou, T. (1994). Consumption-investment models with constraints. SIAM Journal on Control and Optimization, 32(1), 59-85. http://dx.doi.org/10.1137/S0363012991218827 